\title{
Antinormal Weighted Composition Operators
}

\author{
Dilip Kumar and Harish Chandra \\ Department of Mathematics, Banaras Hindu University, Varanasi 221005, India \\ Correspondence should be addressed to Harish Chandra; harishc@bhu.ac.in
}

Received 1 August 2016; Accepted 20 September 2016

Academic Editor: Ngai-Ching Wong

Copyright (C) 2016 D. Kumar and H. Chandra. This is an open access article distributed under the Creative Commons Attribution License, which permits unrestricted use, distribution, and reproduction in any medium, provided the original work is properly cited.

Let $l^{2}=L^{2}(\mathbb{N}, \mu)$, where $\mathbb{N}$ is set of all positive integers and $\mu$ is the counting measure whose $\sigma$-algebra is the power set of $\mathbb{N}$. In this paper, we obtain necessary and sufficient conditions for a weighted composition operator to be antinormal on the Hilbert space $l^{2}$. We also determine a class of antinormal weighted composition operators on Hardy space $H^{2}(\mathbb{D})$.

\section{Introduction}

A deep and interesting problem in operator theory is to determine the distance of an operator from a given class of bounded linear operators on a Hilbert space. In particular, the distance between an operator and the set of Hermitian, positive, compact, and unitary operators has been investigated in [1-4]. In 1974, Holmes [5] discovered that there are operators for which their largest possible distance from the set of normal operators can be achieved. He named such operators as antinormal operators and showed that no compact operator is antinormal. Thus, this extremality of operators is a consequence of infinite dimensional phenomenon of the underlying space. Subsequently, this class has been extensively studied by several authors in [6-9]. For the first time, the same problem has been studied in context of composition operators on the Hilbert space $l^{2}$ in [10] by Tripathi and Lal. In this paper, we investigate antinormality of weighted composition operators on $l^{2}$ and $H^{2}(\mathbb{D})$.

Notation and Terminology. In this paper, $\mathbb{N}$ and $\mathbb{C}$ denote the set of all positive integers and the set of all complex numbers, respectively. Also, let $\chi_{n}: \mathbb{N} \rightarrow\{0,1\}$ be defined as

$$
\chi_{n}(m)= \begin{cases}1, & \text { if } m=n \\ 0, & \text { otherwise. }\end{cases}
$$

Suppose $H$ is a separable complex Hilbert space and $B(H)$ denotes the algebra of all bounded linear operators on $H$. Further, for $T \in B(H)$, let $N(T)$ and $R(T)$, respectively, denote the null space and the range space of $T$.

We now record certain definitions and results which are useful in our context.

Definition 1. A function $u: X \rightarrow \mathbb{C}$ is said to be bounded away from zero if there exists a positive real number, say $r$, such that $0<1 /|u(x)| \leq r$ for all $x \in X$.

Definition 2 (see [11]). An operator $T \in B(H)$ is said to be Fredholm operator if dimension of $N(T)$ and the dimension of the quotient space $H / R(T)$ are both finite.

We also note here that, equivalently, $T$ is Fredholm if both $N(T)$ and $N\left(T^{*}\right)$ are finite dimensional.

Definition 3. Essential spectrum of an operator $T \in B(H)$ is defined as $\sigma_{e}(T)=\{\alpha \in \mathbb{C}: T-\alpha I$ is not Fredholm $\}$.

Since every invertible operator is Fredholm operator, therefore $\sigma_{e}(T) \subseteq \sigma(T)$; see [12].

Definition 4. Minimum modulus of an operator $T \in B(H)$ is defined as $m(T)=\inf \{\|T x\|:\|x\|=1\}$.

Definition 5. Essential minimum modulus of an operator $T \epsilon$ $B(H)$ is defined as $m_{e}(T)=\inf \left\{\alpha \geq 0: \alpha \in \sigma_{e}(|T|)\right\}$, where $|T|=\left(T^{*} T\right)^{1 / 2}$. 
Definition 6. An operator $T \in B(H)$ is said to be antinormal if $d(T, \mathcal{N})=\inf _{N \in \mathcal{N}}\|T-N\|=\|T\|$, where $\mathcal{N}$ is the class of all normal operators in $B(H)$.
Remark 7. An operator $T \in B(H)$ is antinormal if and only if its adjoint $T^{*}$ is antinormal.

Definition 8. For an operator $T$ in $B(H)$, index of $T$ is defined as

if $\operatorname{dim}(N(T))$ or $\operatorname{dim}\left(N\left(T^{*}\right)\right)<\infty$
otherwise.
Remark 9. Observe that index $(T)=-\operatorname{index}\left(T^{*}\right)$.

The following results will be used in the later part of the paper.

Theorem 10 (see [7]). Let $T \in B(H)$.

(i) If index $(T)=0$, then $d(T, \mathcal{N}) \leq(\|T\|-m(T)) / 2$.

(ii) If index $(T)<0$, then $m_{e}(T) \leq d(T, \mathcal{N}) \leq(\|T\|+$ $\left.m_{e}(T)\right) / 2$.

Remark 11. If index $(T)=0$, then $T$ is not antinormal.

Theorem 12 (see [7]). Let $T \in B(H)$ with index $(T)<0$. Then, following conditions are equivalent:

(i) $T$ is antinormal.

(ii) $m_{e}(T)=\|T\|$.

(iii) $d(T, \mathcal{U})=1+\|T\|$, where $\mathcal{U}$ is the class of all unitary operators in $B(H)$.

(iv) $T=\alpha W(1-K)$ for some $\alpha>0$, isometry $W$ and positive compact contraction $K$.

\section{Antinormal Weighted Composition Operators on $l^{2}$}

The Hilbert space $l^{2}$ is the space of all square summable sequences of complex numbers. Let $\varphi: \mathbb{N} \rightarrow \mathbb{N}$ be self-map and $\varphi^{-1}(n)$ denote the inverse image of $n$ under $\varphi$. Let $\left|\varphi^{-1}(n)\right|$ denote the cardinality of $\varphi^{-1}(n)$. For $u \in l^{\infty}$, a weighted composition transformation induced by $u$ and $\varphi$ is defined as

$$
\left(u C_{\varphi}\right)(f)(n)=u(n) f(\varphi(n)) \quad \forall f \in l^{2}, \forall n \in \mathbb{N}
$$

If $u C_{\varphi}$ is bounded, then it is called weighted composition operator induced by $u$ and $\varphi$. The following result gives a necessary and sufficient condition for boundedness of $u C_{\varphi}$.

Theorem 13. Weighted composition operator $u C_{\varphi}$ on $l^{2}$ is bounded if and only if $\sup _{n \in \mathbb{N}}\left\{|u(n)|^{2}\left|\varphi^{-1}(n)\right|\right\}<\infty$. In this case, $\left\|u C_{\varphi}\right\|=\left\{\sup _{n \in \mathbb{N}}\left\{|u(n)|^{2}\left|\varphi^{-1}(n)\right|\right\}\right\}^{1 / 2}$.
Proof. Let $f=\sum_{n \in \mathbb{N}} f(n) \chi_{n}$ be an element in $l^{2}$. Then,

$$
\begin{aligned}
\left\|u C_{\varphi} f\right\|^{2} & =\left\|u C_{\varphi}\left(\sum_{n \in \mathbb{N}} f(n) \chi_{n}\right)\right\|^{2} \\
& =\left\|\sum_{n \in \mathbb{N}} u(n) f(n) \chi_{\varphi^{-1}(n)}\right\|^{2} \\
& =\sum_{n \in \mathbb{N}}|u(n)|^{2}|f(n)|^{2}\left|\varphi^{-1}(n)\right| \\
& \leq \sup _{n \in \mathbb{N}}\left\{|u(n)|^{2}\left|\varphi^{-1}(n)\right|\right\} \sum_{n \in \mathbb{N}}|f(n)|^{2} \\
& =\sup _{n \in \mathbb{N}}\left\{|u(n)|^{2}\left|\varphi^{-1}(n)\right|\right\}\|f\|^{2} .
\end{aligned}
$$

Thus,

$$
\sup _{n \in \mathbb{N}}\left\{|u(n)|^{2}\left|\varphi^{-1}(n)\right|\right\}<\infty
$$

implies that $u C_{\varphi}$ is bounded and

$$
\left\|u C_{\varphi}\right\| \leq\left\{\sup _{n \in \mathbb{N}}\left\{|u(n)|^{2}\left|\varphi^{-1}(n)\right|\right\}\right\}^{1 / 2} .
$$

Conversely, if $u C_{\varphi}$ is bounded, then we have

$$
\left\|u C_{\phi} \chi_{n}\right\|^{2}=|u(n)|^{2}\left|\varphi^{-1}(n)\right| \quad \forall n \in \mathbb{N} .
$$

Therefore,

$$
\left\{\sup _{n \in \mathbb{N}}\left\{|u(n)|^{2}\left|\varphi^{-1}(n)\right|\right\}\right\}^{1 / 2} \leq\left\|u C_{\varphi}\right\|,
$$

hence the proof.

In the following result, we determined weighted composition operators on $l^{2}$ which are not antinormal.

Theorem 14. Let $u \in l^{\infty}$ be bounded away from zero and $\varphi$ be a bijective self-map on $\mathbb{N}$. Then, $u C_{\varphi}$ is not antinormal.

Proof. Since $\varphi$ is bijective and $u$ is bounded away from zero, $u C_{\varphi}$ is invertible on $l^{2}$. Hence, $\operatorname{index}\left(u C_{\varphi}\right)=0$. Consequently, $u C_{\varphi}$ is not antinormal by Remark 11 . 
The following remark shows that the condition that " $u$ is bounded away from zero" is not essential for the validity of the above theorem.

Remark 15. Let $u \in l^{\infty}$ be such that $u(n) \rightarrow 0$ as $n \rightarrow \infty$. Then, it is well known that $u C_{\varphi}$ is compact $([13]$, p. 89). Hence, $u C_{\varphi}$ is not antinormal.

We now prove the following lemma.

Lemma 16. Suppose $\varphi$ is injective but not surjective and $u(n) \neq$ 0 for each $n \in \mathbb{N}$. Then, $u C_{\varphi}$ is not injective but $\left(u C_{\varphi}\right)^{*}$ is injective.

Proof. Since $\varphi$ is not surjective, there exists $n_{0} \in \mathbb{N}$ such that $\varphi^{-1}\left(n_{0}\right)$ is empty. Therefore,

$$
\left(u C_{\varphi}\right) \chi_{n_{0}}=u\left(n_{0}\right) \chi_{\varphi^{-1}\left(n_{0}\right)}=0 .
$$

Consequently, $u C_{\varphi}$ is not injective.

Now, for $f=\sum_{n \in \mathbb{N}} f(n) \chi_{n}$, we have

$$
\left(u C_{\varphi}\right)^{*} f=\sum_{n \in \mathbb{N}} \overline{u(n)} f(n) \chi_{\varphi(n)}
$$

Now, a simple computation using the above expression and injectivity of $\varphi$ shows that whenever $\left(u C_{\varphi}\right)^{*} f=0$, then $\overline{u(n)} f(n)=0 \forall n \in \mathbb{N}$. Since $u(n) \neq 0 \forall n \in \mathbb{N}$, therefore $f=0$. Hence, $\left(u C_{\varphi}\right)^{*}$ is injective.

The following result gives a necessary and sufficient condition for antinormality of $u C_{\varphi}$ when $\varphi$ is injective but not surjective.

Theorem 17. Suppose $u(n) \neq 0$ for each $n \in \mathbb{N}$ and $\varphi$ is injective but not surjective. Then, $u C_{\varphi}$ is antinormal if and only if the following conditions hold:

(a) For each $0 \leq \alpha<\left\|u C_{\varphi}\right\|^{2}, \overline{u(n)} u(\varphi(n)) \neq \alpha$, except for finitely many $n \in \mathbb{N}$.

(b) $\left\|u C_{\varphi}\right\|^{2}=\overline{u(n)} u(\varphi(n))$ for infinitely many $n \in \mathbb{N}$.

Proof. Since $\varphi$ is injective but not surjective and $u(n) \neq 0$ for each $n \in \mathbb{N}$, hence by above lemma index $\left(\left(u C_{\varphi}\right)^{*}\right)<0$. Now, for $f=\sum_{n \in \mathbb{N}} f(n) \chi_{n} \in l^{2}$ and for given complex number $\alpha$, we have

$$
\begin{aligned}
& \left(\left(u C_{\varphi}\right)\left(u C_{\varphi}\right)^{*}-\alpha I\right) f \\
& \quad=\sum_{n \in \mathbb{N}}(\overline{u(n)} u(\varphi(n))-\alpha) f(n) \chi_{n} .
\end{aligned}
$$

From the above equation and condition (a) it follows that $\operatorname{dimker}\left(\left(u C_{\varphi}\right)\left(u C_{\varphi}\right)^{*}-\alpha I\right)=\operatorname{dimker}\left(\left(u C_{\varphi}\right)\left(u C_{\varphi}\right)^{*}-\alpha I\right)^{*}$ is finite whenever $0 \leq \alpha<\left\|u C_{\varphi}\right\|^{2}$. Hence, $\left(\left(u C_{\varphi}\right)\left(u C_{\varphi}\right)^{*}-\right.$ $\alpha I)$ is Fredholm for each $0 \leq \alpha<\left\|u C_{\varphi}\right\|^{2}$. Thus, $\sqrt{\alpha} \notin$ $\sigma_{e}\left(\left|\left(u C_{\varphi}\right)^{*}\right|\right)$ for each $0 \leq \alpha<\left\|u C_{\varphi}\right\|^{2}$. Again by condition (b) $\left\|u C_{\varphi}\right\|^{2}=\overline{u(n)} u(\varphi(n))$ for infinitely many $n \in \mathbb{N}$. Hence, dimker $\left(\left(u C_{\varphi}\right)\left(u C_{\varphi}\right)^{*}-\left\|u C_{\varphi}\right\|^{2} I\right)$ is infinite. Therefore, $\left\|u C_{\varphi}\right\| \in \sigma_{e}\left(\left|\left(u C_{\varphi}\right)^{*}\right|\right)$. Thus, $m_{e}\left(\left(u C_{\varphi}\right)^{*}\right)=\left\|u C_{\varphi}\right\|$. Hence, $\left(u C_{\varphi}\right)^{*}$ is antinormal by Theorem 12 . Now, since adjoint of an antinormal operator is antinormal, therefore $u C_{\varphi}$ is antinormal. Conversely, if either of the conditions fail, then we claim that $u C_{\varphi}$ is not antinormal. For, if condition (a) fails, there exists an $\alpha_{0}$ with $0 \leq \alpha_{0}<\left\|u C_{\varphi}\right\|^{2}$ such that $\sqrt{\alpha_{0}} \in \sigma_{e}\left(\left|\left(u C_{\varphi}\right)^{*}\right|\right)$. Therefore, $m_{e}\left(\left(u C_{\varphi}\right)^{*}\right) \leq \sqrt{\alpha}_{0}<\left\|u C_{\varphi}\right\|$. Hence, $\left(u C_{\varphi}\right)^{*}$ is not antinormal. Now, suppose condition (b) fails. Then, by (11), $\left\|\left(u C_{\varphi}\right)^{*}\right\| \notin \sigma_{e}\left(\left|\left(u C_{\varphi}\right)^{*}\right|\right)$. Therefore, $m_{e}\left(\left(u C_{\varphi}\right)^{*}\right)<\left\|u C_{\varphi}\right\|$. Hence, $u C_{\varphi}$ is not antinormal.

We now prove the following lemma.

Lemma 18. Suppose $\varphi$ is surjective but not injective and $u(n) \neq$ 0 for each $n \in \mathbb{N}$. Then, $u C_{\varphi}$ is injective but $\left(u C_{\varphi}\right)^{*}$ is not injective.

Proof. Let $f \in l^{2}$ be such that $u C_{\varphi}(f)=0$. Therefore, $u(n) f(\varphi(n))=0 \forall n \in \mathbb{N}$. This implies $f(\varphi(n))=0 \forall n \in \mathbb{N}$ as $u(n) \neq 0 \forall n \in \mathbb{N}$. Now, subjectivity $\varphi$ implies $f=0$. Further, Since $\varphi$ is not injective, there exist $m, n \in \mathbb{N}$ with $m \neq n$ such that $\varphi(m)=\varphi(n)$. Now, define $f=\overline{u(m)} \chi_{n}$ and $g=\overline{u(n)} \chi_{m}$. It is easy to see that $f, g \in l^{2}$ and $f \neq g$ but $\left(u C_{\varphi}\right)^{*}(f)=$ $\left(u C_{\varphi}\right)^{*}(g)$. Therefore, $\left(u C_{\varphi}\right)^{*}$ is not injective.

Using above lemma we prove the following result.

Theorem 19. Suppose $u(n) \neq 0$ for each $n \in \mathbb{N}$ and $\varphi$ is surjective but not injective. Then, $u C_{\varphi}$ is antinormal if and only if the following conditions hold:

(a) For each $0 \leq \alpha<\left\|u C_{\varphi}\right\|^{2}, u(n) \sum_{m \in \varphi^{-1}(n)} \overline{u(m)} \neq \alpha$, except for finitely many $n \in \mathbb{N}$,

(b) $\left\|u C_{\varphi}\right\|^{2}=u(n) \sum_{m \in \varphi^{-1}(n)} \overline{u(m)}$ for infinitely many $n \epsilon$ $\mathbb{N}$.

Proof. Since $\varphi$ is surjective but not injective and $u(n) \neq 0$ for each $n \in \mathbb{N}$, hence by above lemma index $\left(\left(u C_{\varphi}\right)^{*}\right)<0$. For $f \in l^{2}$ and $\alpha \in \mathbb{C}$, we have

$$
\begin{aligned}
& \left(\left(u C_{\varphi}\right)^{*}\left(u C_{\varphi}\right)-\alpha I\right) f \\
& \quad=\sum_{n \in \mathbb{N}}\left(u(n) \sum_{m \in \varphi^{-1}(n)} \overline{u(m)}-\alpha\right) f(n) \chi_{n} .
\end{aligned}
$$

Therefore, dimker $\left(\left(u C_{\varphi}\right)^{*}\left(u C_{\varphi}\right)-\alpha I\right)=\operatorname{dimker}\left(\left(u C_{\varphi}\right)^{*}\left(u C_{\varphi}\right)-\right.$ $\alpha I)^{*}$ is finite for each $0 \leq \alpha<\left\|u C_{\varphi}\right\|^{2}$ by condition (a). Hence, $\sqrt{\alpha} \notin \sigma_{e}\left(\left|u C_{\varphi}\right|\right)$ for each $0 \leq \alpha<\left\|u C_{\varphi}\right\|^{2}$. Also, $u(n) \sum_{m \in \varphi^{-1}(n)} \overline{u(m)}=\left\|u C_{\varphi}\right\|^{2}$ for infinitely many $n \in \mathbb{N}$, so $\left\|u C_{\varphi}\right\| \in \sigma_{e}\left(\left|u C_{\varphi}\right|\right)$. Thus, $m_{e}\left(u C_{\varphi}\right)=\left\|u C_{\varphi}\right\|$. Consequently, $u C_{\varphi}$ is antinormal. Conversely, if either of the conditions is not true, then we claim that $u C_{\varphi}$ is not antinormal. If condition (a) fails, then there exists an $\alpha_{0}$ with $0 \leq \alpha_{0}<\left\|u C_{\varphi}\right\|^{2}$, such that $\sqrt{\alpha_{0}} \in \sigma_{e}\left(\left|u C_{\varphi}\right|\right)$. Therefore, $m_{e}\left(u C_{\varphi}\right) \leq \sqrt{\alpha_{0}}<$ $\left\|u C_{\varphi}\right\|$ and so $u C_{\varphi}$ is not antinormal. Now, suppose condition (b) fails. Then, by (12), $\left\|u C_{\varphi}\right\| \notin \sigma_{e}\left(\left|u C_{\varphi}\right|\right)$. Therefore, 
$m_{e}\left(u C_{\varphi}\right)<\left\|u C_{\varphi}\right\|$. Thus, $u C_{\varphi}$ is not antinormal in either case.

Theorem 20. Suppose $u(n) \neq 0 \forall n \in \mathbb{N}$ and $\varphi$ is neither injective nor surjective.

(i) If index $\left(u C_{\varphi}\right)=0$, then $u C_{\varphi}$ is not antinormal.

(ii) If index $\left(u C_{\varphi}\right)<0$, then $u C_{\varphi}$ is antinormal if and only if the following conditions hold:

(a) for each $0 \leq \alpha<\left\|u C_{\varphi}\right\|, u(n) \sum_{m \in \varphi^{-1}(n)} \overline{u(m)} \neq$ $\alpha$, except for finitely many $n \in \operatorname{range}(\varphi)$,

(b) if $\left\|u C_{\varphi}\right\|^{2}=u(n) \sum_{m \in \varphi^{-1}(n)} \overline{u(m)}$ for infinitely many $n \in \operatorname{range}(\varphi)$.

Proof. (i) It follows by Remark 11.

(ii) Proof is same as in case of Theorem 19.

We now give examples, one in each case, satisfying Theorems 17 and 19.

Example 21. Let $\varphi(n)=2 n$ and

$$
u(n)= \begin{cases}1, & n=1,2 \\ 2, & \text { otherwise. }\end{cases}
$$

Clearly, $\varphi$ is injective but not surjective and $\overline{u(n)} u(\varphi(n))=4=$ $\left\|u C_{\varphi}\right\|^{2}$ for $n \geq 2$. Hence, by Theorem $17, u C_{\varphi}$ is antinormal.

Example 22. Let

$$
\begin{aligned}
& \varphi(n)= \begin{cases}\frac{n+1}{2}, & n \text { is odd } \\
\frac{n}{2}, & n \text { is even, }\end{cases} \\
& u(n)= \begin{cases}1, & n=1 \\
2, & \text { otherwise. }\end{cases}
\end{aligned}
$$

Clearly, $\varphi$ is surjective but not injective and $\left\|u C_{\varphi}\right\|^{2}=8=$ $u(n) \sum_{m \in \varphi^{-1}(n)} \overline{u(m)}$ for $n \geq 2$. Hence, antinormality of $u C_{\varphi}$ follows from Theorem 19.

\section{Antinormal Weighted Composition Operators on Hardy Space}

In this section, we continue our line of investigation of antinormal operators. Let $\mathbb{D}=\{z \in \mathbb{C}:|z|<1\}$ and $\partial \mathbb{D}=\{z \in$ $\mathbb{C}:|z|=1\}$. Hardy space $H^{2}(\mathbb{D})[14]$ is defined to be the space of all analytic functions $f$ on the unit disk $\mathbb{D}$ with the property

$$
\|f\|^{2}=\sup _{0 \leq r<1} \int_{0}^{2 \pi}|f(r z)|^{2} \frac{d \theta}{2 \pi}<\infty .
$$

The inner product on $H^{2}(\mathbb{D})$ is defined as follows:

$$
\langle f, g\rangle=\int_{0}^{2 \pi} f\left(e^{i \theta}\right) \overline{g\left(e^{i \theta}\right)} \frac{d \theta}{2 \pi} .
$$

Let $\varphi: \mathbb{D} \rightarrow \mathbb{D}$ be analytic self-map. For $u \in H^{\infty}(\mathbb{D})$, weighted composition transformation $u C_{\varphi}$ on $H^{2}(\mathbb{D})$ is defined by $\left(u C_{\varphi}\right)(f)(z)=u(z) f(\varphi(z))$ for all $f \in H^{2}(\mathbb{D})$ and for all $z \in \mathbb{D}$. The fact that $u C_{\varphi}$ is bounded linear operator on $H^{2}(\mathbb{D})$ follows from the well-known Hardy-Littlewood subordination, Theorem [15]. In 2011, Gunatillake [16] has shown that the operator $u C_{\varphi}$ on $H^{2}(\mathbb{D})$ is invertible if and only if $u$ is both bounded and bounded away from zero on the unit disc and $\varphi$ is an automorphism of the unit disc.

Following result is an easy consequence of preceding statement.

Theorem 23. If $u \in H^{\infty}(\mathbb{D})$ is bounded away from zero and $\varphi$ is automorphism of the unit disc, then $u C_{\varphi}$ is not an antinormal composition operator.

Proof. Since $u C_{\varphi}$ is bijective, so index $\left(u C_{\varphi}\right)=0$. Hence, $C_{\varphi}$ is not antinormal by Remark 11.

One of the difficult issues is to find a useful description of the adjoint of $u C_{\varphi}$ for arbitrary $\varphi$. In 2011, Matache [17] showed that if $\varphi(0)=0$ then $C_{\varphi}^{*}\left(z^{n}\right)=\sum_{k=1}^{k=n}\left\langle z^{n}, \varphi^{k}\right\rangle z^{k}$ for each $n=1,2,3, \ldots$. We have used his idea in the following theorem.

Theorem 24. If self-map $\varphi(z)=z^{n}$ for $n \geq 2$ and $|u(z)|=1$ a.e. on $\partial \mathbb{D}$, then $u C_{\varphi}$ is antinormal.

Proof. Let $f(z)=\sum_{r \geq 0} a_{r} z^{r} \in H^{2}(\mathbb{D})$. Then,

$$
\begin{aligned}
& \left(u C_{\varphi}\right)^{*}\left(u C_{\varphi}\right) f(z)=\sum_{r \geq 0} a_{r}\left(u C_{\varphi}\right)^{*}\left(u C_{\varphi}\right)\left(z^{r}\right) \\
& =\sum_{r \geq 0} a_{r}\left(u C_{\varphi}\right)^{*}\left(u(z) \varphi^{r}(z)\right) \\
& =\sum_{r \geq 0} a_{r} \sum_{k \geq 0}\left\langle u C_{\varphi}\right)^{*}\left(u(z) \varphi^{r}(z), z^{k}\right\rangle z^{k} \\
& =\sum_{r \geq 0} a_{r} \sum_{k \geq 0}\left\langle u(z) \varphi^{r}(z), u C_{\varphi}\left(z^{k}\right)\right\rangle z^{k} \\
& =\sum_{r \geq 0} a_{r} \sum_{k \geq 0}\left\langle u(z) \varphi^{r}(z), u(z) \varphi^{k}(z)\right\rangle z^{k} \\
& =\sum_{r \geq 0} a_{r} \sum_{k \geq 0}\left(\int_{\partial \mathbb{D}} u\left(e^{i \theta}\right) \varphi^{r}\left(e^{i \theta}\right) \frac{u\left(e^{i \theta}\right) \varphi^{k}\left(e^{i \theta}\right)}{2 \pi}\right) z^{k} \\
& =\sum_{r \geq 0} a_{r} \sum_{k \geq 0}\left(\int_{\partial \mathbb{D}} e^{i n r \theta} e^{-i n k \theta} \frac{d \theta}{2 \pi}\right) z^{k}=\sum_{r \geq 0} a_{r} z^{r}=f(z) .
\end{aligned}
$$

Therefore $\left(u C_{\varphi}\right)^{*}\left(u C_{\varphi}\right)-\alpha I=(1-\alpha) I \forall \alpha \geq 0$. This implies that $\left(u C_{\varphi}\right)^{*}\left(u C_{\varphi}\right)-\alpha I$ is invertible for $0 \leq \alpha<1$. Hence, $\left(u C_{\varphi}\right)^{*}\left(u C_{\varphi}\right)-\alpha I$ is Fredholm for $0 \leq \alpha<1$. Thus, $\alpha \notin$ $\sigma_{e}\left(\left|u C_{\varphi}\right|\right)$ whenever $0 \leq \alpha<1$. Also $\operatorname{dimker}\left(\left(u C_{\varphi}\right)^{*}\left(u C_{\varphi}\right)-\right.$ $\alpha I)$ is infinite for $\alpha=1$. Hence, $1 \in \sigma_{e}\left(\left|u C_{\varphi}\right|\right)$. Therefore, $m_{e}\left(u C_{\varphi}\right)=\inf \left\{\alpha \geq 0: \alpha \in \sigma_{e}\left(\left|u C_{\varphi}\right|\right)\right\}=1$. Since $\varphi(0)=0$ and $|u(z)|=1$ a.e. on $\partial \mathbb{D},\left\|u C_{\phi}\right\|=\left\|C_{\varphi}\right\|=1$. Further, $u C_{\varphi}$ is injective, and $u C_{\varphi}$ is not surjective as $\varphi$ is not 
an automorphism. Consequently, index $\left(u C_{\varphi}\right)<0$. Hence, the antinormality of $u C_{\varphi}$ follows from Theorem 12 .

Following corollary is an immediate consequence of the preceding theorem.

Corollary 25. If self-map $\varphi(z)=z^{n} \forall z \in \mathbb{D}, n \geq 2$, then $C_{\varphi}$ is antinormal.

\section{Competing Interests}

The authors declare that they have no competing interests.

\section{References}

[1] K. Fan and A. J. Hoffman, "Some metric inequalities in the space of matrices," Proceedings of the American Mathematical Society, vol. 6, pp. 111-116, 1955.

[2] A. F. ter Elst, "Antinormal operators," Acta Scientiarum Mathematicarum, vol. 54, no. 1-2, pp. 151-158, 1990.

[3] P. R. Halmos, "Positive approximants of operators," Indiana University Mathematics Journal, vol. 21, no. 10, pp. 951-960, 1972.

[4] R. B. Holmes and B. R. Kripke, "Best approximation by compact operators," Indiana University Mathematics Journal, vol. 21, pp. 255-263, 1972.

[5] R. B. Holmes, "Best approximation by normal operators," Journal of Approximation Theory, vol. 12, pp. 412-417, 1974.

[6] D. D. Rogers, "On proximinal sets of normal operators," Proceedings of the American Mathematical Society, vol. 61, no. 1, pp. 44-48, 1976.

[7] S. Izumino, "Inequalities on normal and antinormal operators," Mathematica Japonica, vol. 23, no. 2, pp. 211-215, 1978.

[8] M. Fujii and R. Nakamoto, "Antinormal operators and theorems of Izumino," Mathematica Japonica, vol. 24, no. 1, pp. 41-44, 1979.

[9] S. Izumino, "Inequalities on nilpotent operators," Mathematica Japonica, vol. 24, no. 1, pp. 31-34, 1979.

[10] G. P. Tripathi and N. Lal, "Antinormal composition operators on $\ell^{2}$," Tamkang Journal of Mathematics, vol. 39, pp. 347-352, 2008.

[11] Y. A. Abramovich and C. D. Aliprantis, An Invitation to Operator Theory, vol. 50 of Graduate Studies in Mathematics, American Mathematical Society, 2002.

[12] J. B. Conway, A Course in Functional Analysis, vol. 96 of Graduate Texts in Mathematics, Springer, New York, NY, USA, 2nd edition, 1990.

[13] R. K. Singh and J. S. Manhas, Composition Operators on Function Spaces, vol. 179, North-Holland, New York, NY, USA, 1993.

[14] P. L. Duren, Theory of $H_{p}$ Spaces, vol. 38 of Pure and Applied Mathematics, Academic Press, New York, NY, USA, 1970.

[15] J. H. Shapiro, Composition Operators and Classical Function Theory, Springer, New York, NY, USA, 1993.

[16] G. Gunatillake, "Invertible weighted composition operators," Journal of Functional Analysis, vol. 261, no. 3, pp. 831-860, 2011.

[17] V. Matache, "Composition operators whose symbols have orthogonal powers," Houston Journal of Mathematics, vol. 37, no. 3, pp. 845-857, 2011. 


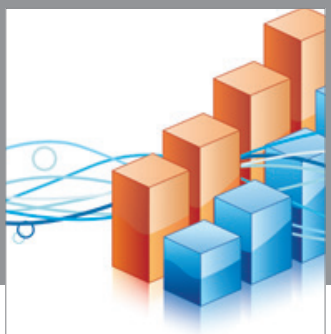

Advances in

Operations Research

vatem alat4

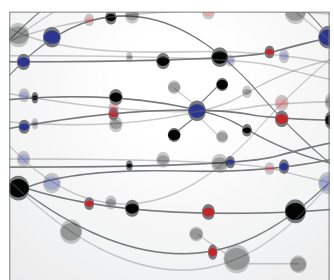

\section{The Scientific} World Journal
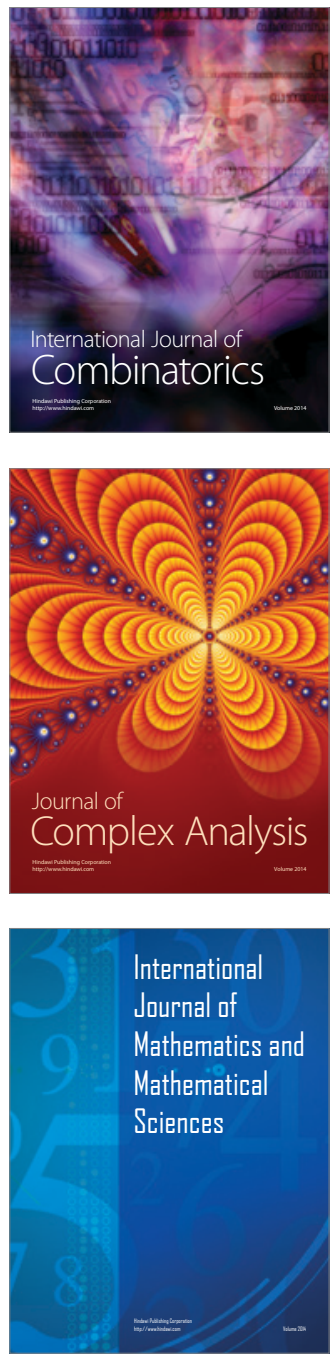
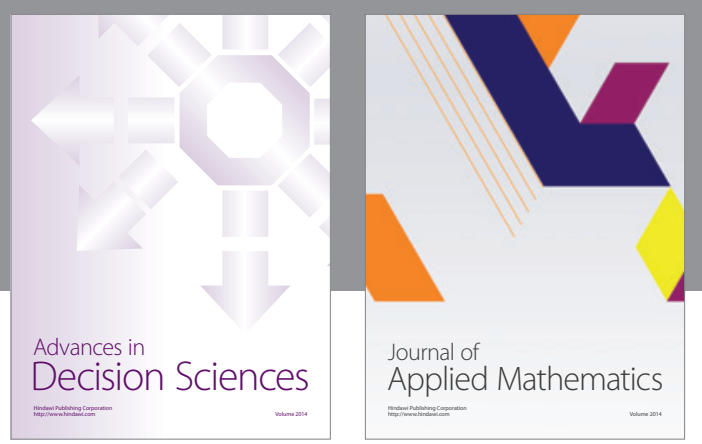

Algebra

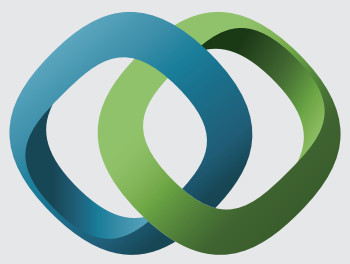

\section{Hindawi}

Submit your manuscripts at

http://www.hindawi.com
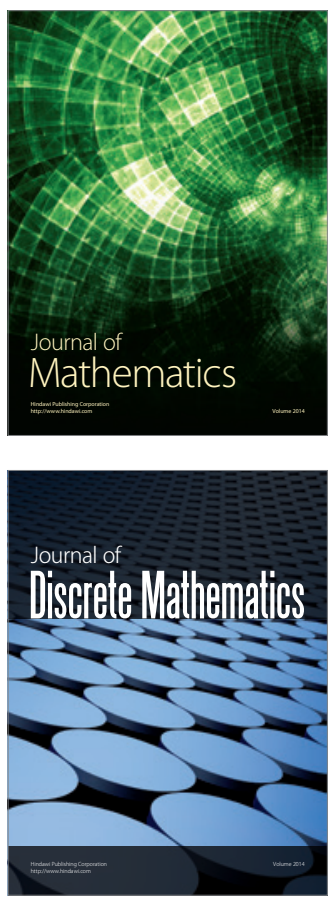

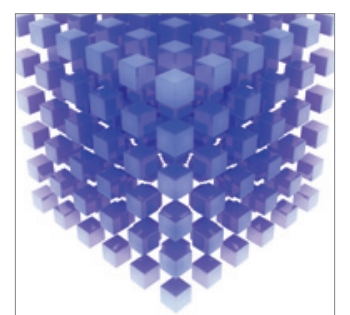

Mathematical Problems in Engineering
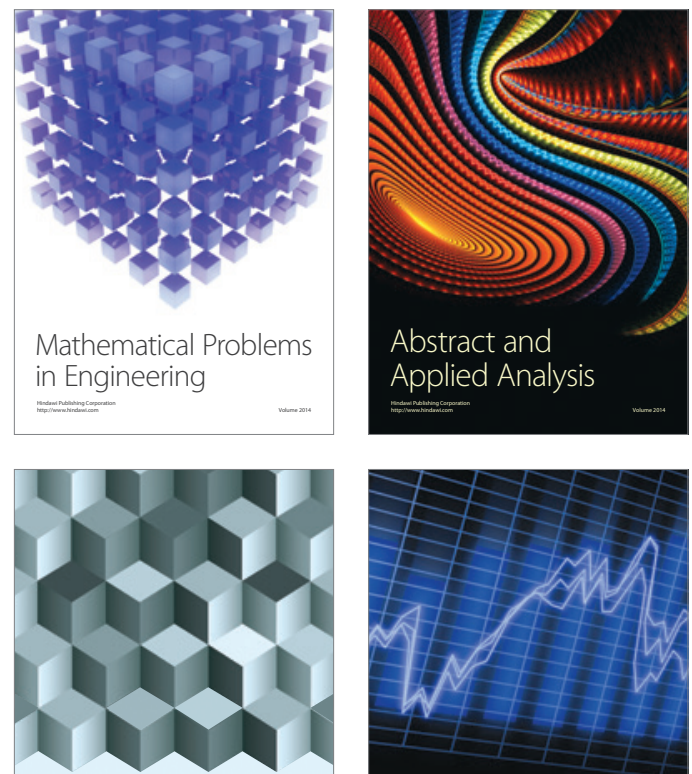

Journal of

Function Spaces

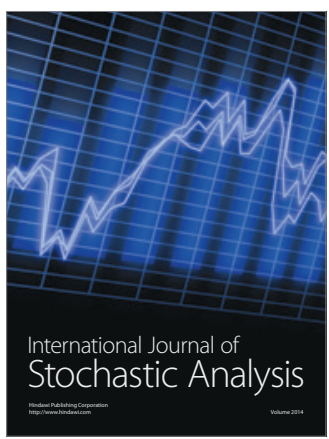

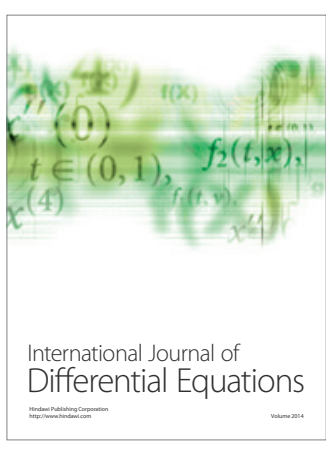
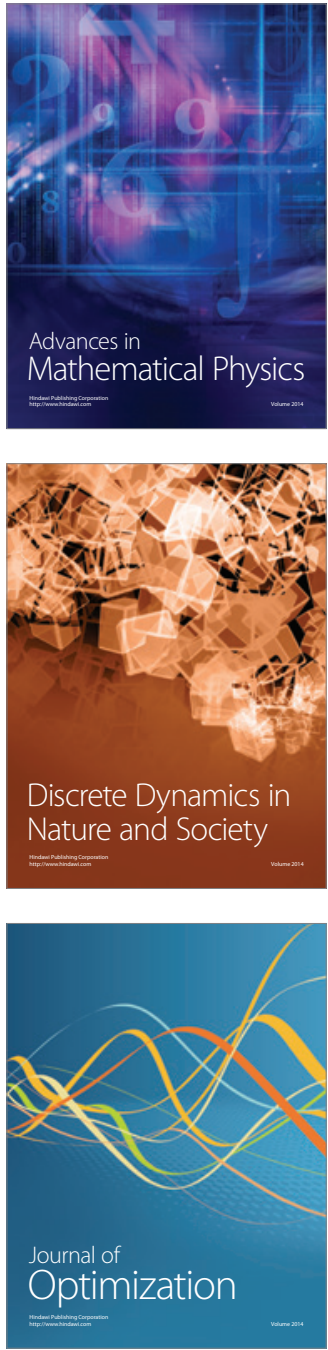